\title{
BIOHEAT EQUATION WITH FOURIER AND NON-FOURIER HEAT TRANSPORT LAWS: APPLICABILITY TO HEAT TRANSFER IN HUMAN TISSUES
}

\author{
N. Kizilova ${ }^{1,2,{ }^{*}}$, A. Korobov ${ }^{3}$
}

\begin{abstract}
The paper is dedicated to mathematical problem formulations for the heat propagation in biological tissues based on the Fourier and non-Fourier laws at different boundary conditions. The heating of the tissues is provided by external heat sources like low intensity lasers or light-emitting diodes which are widely used in contemporary medical care. Numerical computations on the standard Pennes bioheat equation with Fourier heat conduction give the temperature curves for both heating and thermal relaxation processes that do not correspond to the in vivo measurement data on human skin tissue. It is shown the modified bioheat equation based on the Guyer-Krumhansl heat conduction with correct formulation of the boundary conditions produces realistic temperature curves when the distributed heat sources and sinks in the tissue are accounted for. The former corresponds to the metabolic heat and temperature dependent chemical reactions, while the latter is provided by the heat convection with blood microcirculation system. The proposed model gives realistic two time temperature curves. The perspective applications of the novel mathematical formulation are discussed.
\end{abstract}

Keywords: Pennes Bioheat Equation, Non-Fourier Heat Transport Laws, Heat Transfer in Biological Tissues, Mathematical Modeling

\section{INTRODUCTION}

During the last decades different methods of phototherapy and photodynamic therapy based on the coherent optical radiation of low-level lasers (LLL) and non-coherent radiation of light-emitting diodes (LED) had been introduced, tested and approved for routine treatment purposes [1-3]. Optical radiation improves local microcirculation in the tissues, produce stable dilatation of the blood and lymphatic vessels, and produce antiinflammatory, analgesic and lymph-depleting effects [4]. Personal-specific application of the therapy needs detailed quantitative estimations of the time, intensity and dose of the irradiation. For the surface or internal (i.e. via optic fibers or during an open surgery) radiation the correspondent models of the tissues accounting for their individual geometry, dimensions and material parameters are needed. The multilayer models of human skin have been developed for the LLL treatment [5-7]. Similar approaches have been proposed in dentistry [8] and general surgery [9]. Such modeling is based on the heat generation and propagation in the biological tissues due to the photoinduced biochemical reactions and the correspondent variations in the local blood microcirculation.

The very first heat balance equation for biological tissues has been formulated by H. Pennes [10]. It is a heat balance equation accounted for the metabolic heat sources $q_{m e t}$ and heat exchange with the blood microcirculation. The latter is modeled as continuously distributed sources with intensities proportional to the local temperature differences between the blood and tissue. The Pennes bioheat equation has the form

$$
\rho c \frac{\partial T}{\partial t}=\nabla \cdot(k \nabla T)+q_{m e t}+q_{r a d}-\rho_{b} c_{b} w_{b}\left(\mathrm{~T}-T_{b}\right)
$$

This paper was recommended for publication in revised form by Regional Editor N. Filiz Özdil

${ }^{1}$ Warsaw University of Technology, Warsaw, Poland

${ }^{2}$ V.N. Karazin Kharkov National University, Faculty of Mathematics and Informatics, Kharkov, Ukraine

${ }^{3}$ Kharkov National University, Faculty of Radiophysics, Biomedical Electronics and Computer Systems, Ukraine

${ }^{*}$ E-mail address: n.kizilova@gmail.com

Orcid id: 0000-0001-9981-7616,0000-0001-8237-7159

Manuscript Received 1 March 2019, Accepted 25 May 2019 
Journal of Thermal Engineering, Research Article, Vol. 5, No. 6, Special Issue 10, pp. 149-161, December, 2019

where $T$ is the temperature, $\rho, c, k$ are the density, specific heat and thermal conductivity of the tissue, the subscript $b$ refers to the blood, $w_{b}$ is the local blood perfusion rate in the tissue, $q_{\text {rad }}$ represents additional heat sources activated by the irradiation.

The Pennes equation (1) has been used in a series of studies on the local heating or cooling of surface tissues, including the hyperthermia treatment of skin and underlying tissues by LED and LLL [7, 11]. The schema of the experiment and the layers of the surface tissues are shown in Figure 1. For the 1d case the analytical solutions of (1) can be easily obtained at different boundary conditions [12-15]. The corresponding solutions are given by quite cumbersome formulae and are not reproduced here for brevity. For the metabolic heat term the constant value corresponded to the average metabolic rate in the tissues $q_{\text {met }}=$ const,$q_{\text {rad }}=0$ have been mostly used. The typical surface temperature $T(t)$ curves for the heating and relaxation processes computed on the Pennes equation in this case can be found in Figure 2 that present the model validation curves. Similar dynamics have been detected for other types of the heat sources and boundary conditions [7]. Experimental studies on the infrared heating, LED irradiation or/and local cooling of the surface tissues produce dynamics $[14,15]$ which differs from the one predicted by the Pennes equation. For example, the local heating $(20 \mathrm{~min})$ followed by thermal relaxation $(30 \mathrm{~min})$ exhibited complex dynamics of the temperature rise during the heating and clear two time decay relaxation process (Figure 2), while the theoretical estimations based on the Pennes bioheat equation posses simple monotonous temperature rise during the heating and exponential decay during the heat relaxation (see [7]).

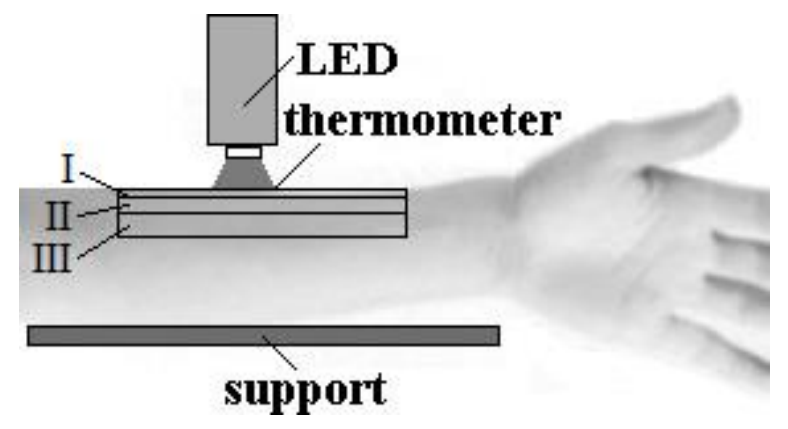

Figure 1. Schema of the surface heating experiment and three layer model of the surface tissues: dermis (I), hypodermis (II), muscle (III)

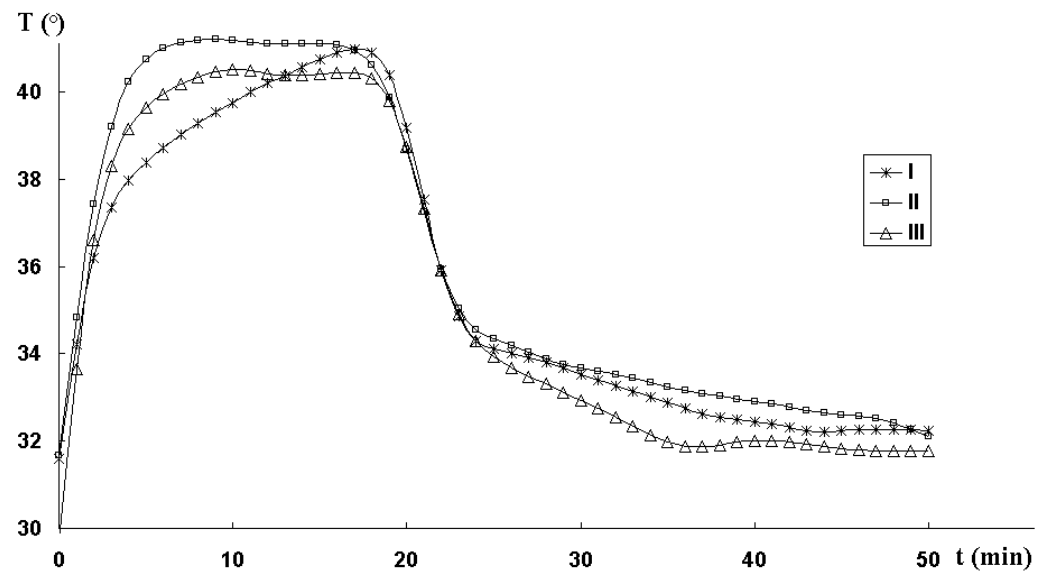

Figure 2. Temperature curves measured for the local infrared radiation $(\mathrm{t}=20 \mathrm{~min})$ followed by thermal relaxation (30 min) over human skin (I), muscle (II) and bone (III) [15]

Different non-Fourier heat transport equations for the micro/nano scale systems, fast processes and complex biological systems with phase transitions at the interfaces have been proposed. The single phase lag (SFL) model introduces a time delay between the local heat flux $q$ and the temperature gradient $\nabla T$ in the form [16,17] 
Journal of Thermal Engineering, Research Article, Vol. 5, No. 6, Special Issue 10, pp. 149-161, December, 2019

$$
q\left(t+\tau_{q}, \vec{r}\right)=-k \nabla T(t, \vec{r})
$$

where $\tau_{q}$ is the heat relaxation time.

Then the bioheat equation (1) has the extended form

$$
\rho c \tau_{q} \frac{\partial^{2} T}{\partial t^{2}}+\left(\rho c+\rho_{b} c_{b} w_{b} \tau_{q}\right) \frac{\partial T}{\partial t}+\rho_{b} c_{b} w_{b} T=\nabla \cdot(k \nabla T)+q_{m e t}+q_{r a d}+\rho_{b} c_{b} w_{b} T_{b}
$$

Unlike the parabolic equation (1), hyperbolic equation (3) describes the thermal wave expansion at a constant speed. When $w_{b}=0, q_{\text {met }}=0, q_{\text {rad }}=0$, (3) gives the Cattaneo-Vernotte model [18,19] of the temperature balance equation.

Additional approach for the temperature gradient relaxation time $\tau_{T}$ has been proposed in the form of the dual phase lag (DFL) model

$$
q\left(t+\tau_{q}, \vec{r}\right)=-k \nabla T\left(t+\tau_{T}, \vec{r}\right)
$$

where the relaxation times $\tau_{T}$ and $\tau_{q}$ are not interconnected.

That approach gives the bioheat DFL model in the form $[6,20]$

$$
\rho c \tau_{q} \frac{\partial^{2} T}{\partial t^{2}}+\left(\rho c+\rho_{b} c_{b} w_{b} \tau_{q}\right) \frac{\partial T}{\partial t}+\rho_{b} c_{b} w_{b} T=k\left(\nabla^{2} T+\tau_{T} \frac{\partial\left(\nabla^{2} T\right)}{\partial t}\right)+q_{m e t}+q_{r a d}+\rho_{b} c_{b} w_{b} T_{b}
$$

When $w_{b}=0, q_{\text {met }}=0, q_{\text {rad }}=0$, (5) gives the Guyer-Krumhansl (GK) equation that is widely used now in the heat transfer studies at the micro/nano scales, but despite the DFL, in the GK model the positive parameter $\kappa^{2}=k \tau_{T}$ is related to the characteristic length scale $\kappa$ and corresponds to the ballistic heat conduction mechanism.

The solutions of the modified bioheat equations (3), (5) with different boundary conditions have been found in analytical, semi-analytical and numerical forms. Theoretical results have been compared to the Pennes equation based computations for the skin heating problem [6, 11-13, 20]. It was found the three models predict similar $T(t)$ dynamics at relatively low values for $\tau_{q}$ and $\tau_{T}$ while at $\tau_{q, T}>10 \mathrm{~s}$ significant differences between the models have been detected.

The triple time lag and higher order equations can not improve the model because they are inconsistent with the second law of thermodynamics [21-23]. The SFL destroys the time translation invariance of the equation, while the DPL model cannot provide a general description of the heat conduction phenomena. The mathematical conditions to obtain an exponentially stable equilibrium solution of DPL model are beyond the physical limits [21,22,24]. Those conditions require negative time delay $\tau_{q}-\tau_{T} \leq 0$ between the heat flux and temperature gradient (retarded effect), and the DPL theory is based on the cause-effect concept, i.e. the quantity with the higher relaxation time is the effect caused by another quantity. That is why the basic model for treatment of the experimental curves and analysis of the regularities in the heat propagation in tissues must be corrected based on a thermodynamically consistent model able to describe the experimentally observed two-time heat relaxation (Figure 2) in the tissues.

The heat conduction law must produce the corresponding heat transfer equation which is in agreement with the second law of thermodynamics and with space-time evolution. The correct thermodynamically consisted nonFourier heat conduction law is the GK equation [24,25] 


$$
\tau \partial_{t} \vec{J}+\vec{J}=-k \nabla T+\kappa^{2} \Delta T
$$

where $\tau$ is the relaxation time, $\kappa$ is the dissipation parameter determined by the free path $\lambda, \kappa \sim \lambda$.

Despite the DFL model, all the parameters in the model (6) are nonnegative and have clear physical meaning. In this work the modified Pennes bioheat equation based on the GK law of heat propagation is studied in application to the experimental data measured on LED irradiation of human skin and correct estimation of the temperature related effects.

\section{PROBLEM FORMULATION FOR THE PENNES BIOHEAT EQUATION WITH GUYER-KRUMHANSL LAW}

Substitution of (6) into the general heat conduction equation gives the following modification of the Pennes bioheat equation

$$
\begin{aligned}
& \tau \frac{\partial^{2} T}{\partial t^{2}}+\frac{\partial T}{\partial t}\left(1+\tau \tilde{\rho} \tilde{c} w_{b}\right)=\left(\alpha-\kappa^{2} \tilde{\rho} \tilde{c} w_{b}\right) \Delta T+\kappa^{2}\left(\frac{\partial}{\partial t} \Delta T\right)-\tilde{\rho} \tilde{c} w_{b}\left(T-T_{b}\right)+ \\
& +\tilde{q}_{m e t}+\tilde{q}_{r a d}+\tau \frac{\partial}{\partial t}\left(\tilde{q}_{m e t}+\tilde{q}_{r a d}\right)-\kappa^{2}\left(\Delta \tilde{q}_{m e t}+\Delta \tilde{q}_{r a d}\right)
\end{aligned}
$$

where $\alpha=k / \rho c$ is the thermal diffusivity, $\tilde{\rho}=\rho / \rho_{b}, \tilde{c}=c / c_{b}, \tilde{q}_{m e t}=q_{\text {met }} / \rho c, \tilde{q}_{\text {rad }}=q_{\text {rad }} / \rho c$.

Since the heat sources $\tilde{q}_{m e t}, \tilde{q}_{\text {rad }}$ include the radiation induced temperature dependent exothermal biochemical reactions, let us assume the tissues are uniform and $\tilde{q}_{m e t}=\tilde{q}_{m e t}(\mathrm{~T}), \tilde{q}_{r a d}=\tilde{q}_{r a d}(T)$. Within a small interval of natural surface temperatures of human skin $\mathrm{T}=T_{0}(1 \pm \varepsilon), \varepsilon \leq 1$ the following linear approximations can be assumed

$$
\tilde{q}_{m e t}=\tilde{q}_{m e t}^{0}+\lambda_{\text {met }}\left(\mathrm{T}-\mathrm{T}_{s}\right), \tilde{q}_{r a d}=\tilde{q}_{r a d}^{0}+\lambda_{\text {rad }}\left(\mathrm{T}-\mathrm{T}_{s}\right)
$$

where $\tilde{q}_{\text {met }}^{s}=\tilde{q}_{\text {met }}\left(\mathrm{T}_{s}\right), \tilde{q}_{\text {rad }}^{s}=\tilde{q}_{\text {rad }}\left(T_{s}\right), \lambda_{\text {met }}, \lambda_{\text {rad }}=$ const,$T_{s}$ is the characteristic steady temperature [1]. In the considered experimental conditions (Figure 2) the measured temperature changed as $T=37 \pm 5^{\circ} C, \varepsilon=0.13$ and the approximations (8) are reasonable.

Then, assuming $T_{b}=$ const (7) can be presented in the form

$$
\tau \frac{\partial^{2} T}{\partial t^{2}}+\mathrm{a}_{1} \frac{\partial T}{\partial t}-\kappa^{2} \frac{\partial}{\partial t} \Delta T-\mathrm{a}_{2} \Delta T+\mathrm{a}_{3} T=\mathrm{a}_{4}
$$

where

$$
\mathrm{a}_{1}=1-\tau\left(\tilde{\rho} \tilde{c} w_{b}+\lambda_{\text {met }}+\lambda_{\text {rad }}\right),
$$

$$
\mathrm{a}_{2}=\alpha-\kappa^{2} \tilde{\rho} \tilde{c} w_{b}
$$

$\mathrm{a}_{3}=\tilde{\rho} \tilde{c} w_{b}-\left(\lambda_{\text {met }}+\lambda_{\text {rad }}\right)$, $\mathrm{a}_{4}=\tilde{\rho} \tilde{c} w_{b} T_{b}+\tilde{q}_{\text {met }}^{s}+\tilde{q}_{\text {rad }}^{s}-\mathrm{T}_{s}\left(\lambda_{\text {met }}+\lambda_{\text {rad }}\right)$, and the stroke sign means derivative with respect to temperature.

In general case (9) is the nonlinear PDE of the second order on time and space with mixed derivatives. Assuming (8) and $\tilde{q}_{m e t}^{\prime}=\lambda_{\text {met }}, \tilde{q}_{\text {rad }}^{\prime}=\lambda_{\text {rad }}, \tilde{q}_{m e t}^{\prime \prime}=0, \tilde{q}_{\text {rad }}^{\prime \prime}=0$, (9) can be rewritten in the form

$$
\mathrm{a}_{1}\left(\frac{\partial T}{\partial t}-\frac{\mathrm{a}_{2}}{\mathrm{a}_{1}} \Delta T\right)+\tau \frac{\partial}{\partial t}\left(\frac{\partial T}{\partial t}-\frac{\kappa^{2}}{\tau} \Delta T\right)=\mathrm{a}_{3} T-\mathrm{a}_{4}
$$

When $\mathrm{a}_{2} / \mathrm{a}_{1}=\kappa^{2} / \tau$, (10) has the form

$$
\left[\mathrm{a}_{1}+\tau \frac{\partial}{\partial t}\right]\left(\frac{\partial T}{\partial t}-\frac{\kappa^{2}}{\tau} \Delta T\right)=\mathrm{a}_{3} T-\mathrm{a}_{4}
$$


where the square brackets are related to the differential operator.

The standard bioheat equation (1) rewritten with new designations is

$$
\frac{\partial T}{\partial t}-\alpha \Delta T=\mathrm{a}_{3} T-\mathrm{a}_{4}
$$

When $\kappa^{2} / \tau=\alpha$, solutions of the classical GK (11) and Fourier heat conduction (12) equations are the same, and this case is called Fourier resonance condition [25]. In the case of the bioheat equation, the Fourier resonance condition $\mathrm{a}_{2} / \mathrm{a}_{1}=\alpha$ can be provided by the balance between the source terms in the form

$$
\tilde{q}_{m e t}^{\prime \prime}-\tilde{q}_{m e t}^{\prime}=-\left(\tilde{q}_{r a d}^{\prime \prime}-\tilde{q}_{r a d}^{\prime}\right)
$$

or, accounting for (8), $\lambda_{\text {met }}+\lambda_{\text {rad }}=0$. Since $\lambda_{\text {met }}>0$, the resonance condition is possible in the specific case when $\lambda_{\text {met }}=-\lambda_{\text {rad }}<0$, i.e. for the frequencies inducing the endothermal biochemical reactions [4].

When $\mathrm{a}_{2} / \mathrm{a}_{1}>\kappa^{2} / \tau$, solution of (11) is overdiffusive [25,26]. For the classical GK equation, it means the Peclet number $P e=\kappa^{2} / \tau \alpha>1$ and convective heat conduction prevails over the molecular one. For the bioheat equation the condition is satisfied when $\left(\tilde{q}_{m e t}^{\prime \prime}-\tilde{q}_{m e t}^{\prime}\right)+\left(\tilde{q}_{r a d}^{\prime \prime}-\tilde{q}_{r a d}^{\prime}\right)<0$. The latter can be satisfied when, for instance, both metabolic and photoinduced reactions exhibit exponential growth with temperature.

\section{ONE-DIMENSIONAL HEAT PROPAGATION PROBLEM FOR BIOLOGICAL MATERIAL}

Let us consider one-dimensional heat propagation across the uniform layer of biological tissue $0<x<h$ (Figure 3). The material is irradiated by LLL, LED or other source of heat at the surface $x=0$ and the external heat flux $\vec{J}_{1}(t)$ is known. At the surface $x=h$ another heat flux $\vec{J}_{2}(t)$ is assumed to be given. Since in the biological tissues the surface $x=h$ is deep located and its temperature distribution cannot be measured directly, it is natural to assume $\vec{J}_{2}(t)=0$ and determine $h$ as a penetration depth for the irradiation source applied. Here the solution will be found for the more general case $\vec{J}_{2}(t) \neq 0$

$$
\begin{aligned}
& x=0:-\lambda \frac{\partial T}{\partial x}=J_{1}(t) \\
& x=h:-\lambda \frac{\partial T}{\partial x}=J_{2}(t)
\end{aligned}
$$

At $t=0$ the temperature distribution in the tissues is supposed to be correspondent to the temperature of blood

$$
t=0: \quad T=T_{b}
$$

In the non-dimensional variables $T^{\circ}=\left(T-T_{b}\right) / T^{*}, x^{\circ}=x / h, t^{\circ}=t / t^{*}$ the equations (1), (3), (9) and boundary conditions (14)-(16) (the symbols ${ }^{\circ}$ at the variables are omitted for simplicity) read

$$
\frac{\partial T}{\partial t}=\alpha^{0} \frac{\partial^{2} T}{\partial x^{2}}-\chi T+q
$$


Journal of Thermal Engineering, Research Article, Vol. 5, No. 6, Special Issue 10, pp. 149-161, December, 2019

$$
\begin{gathered}
\tau^{0} \frac{\partial^{2} T}{\partial t^{2}}+\left(1+\chi \tau^{0}\right) \frac{\partial T}{\partial t}=\alpha^{0} \frac{\partial^{2} T}{\partial x^{2}}-\chi T+q \\
\tau^{0} \frac{\partial^{2} T}{\partial t^{2}}+\left(1+\tau^{0}\left(\chi-q^{\prime}\right)\right) \frac{\partial T}{\partial t}=K n^{2} \frac{\partial^{3} T}{\partial t \partial x^{2}}+\left(\alpha^{0}-K n^{2} \chi\right) \frac{\partial^{2} T}{\partial x^{2}}-\left(\chi-q^{\prime}\right) T+q \\
x=0: \frac{\partial T}{\partial x}=J_{1}^{0}(t) \\
x=1: \frac{\partial T}{\partial x}=J_{2}^{0}(t) \\
t=0: \quad T=0
\end{gathered}
$$

where $\quad \alpha^{0}=\alpha t^{*} / h^{2}, \quad w_{b}^{0}=w_{b} t^{*}, \quad \tau^{0}=\tau / t^{*}, \quad \chi=\tilde{\rho} \tilde{c} w_{b}^{0}, \quad q=q_{\text {met }}^{0}+q_{\text {rad }}^{0}-\mathrm{T}_{s}^{0}\left(\lambda_{\text {met }}^{0}+\lambda_{\text {rad }}^{0}\right), \quad q^{\prime}=\lambda_{\text {met }}^{0}+\lambda_{\text {rad }}^{0}$, $q_{\text {met,rad }}^{0}=\tilde{q}_{\text {met,rad }}^{s}{ }^{*}, \mathrm{~T}_{s}^{\circ}=\mathrm{T}_{s} / T^{*}, \lambda_{\text {met }, \text { rad }}^{0}=\lambda_{\text {met, }, \text { ad }} / \rho c, J_{1,2}^{0}(t)=J_{1,2}(t) \mathrm{h} / \lambda \mathrm{T}^{*}, K n=\kappa / h$ is the Knudsen number. Generally speaking, the equivalent $\mathrm{T}_{s}=\mathrm{T}_{b}$ may be accepted, but in this study both temperatures are taken as different.

The terms $\sim K n^{2}$ are essential for the nanostructural materials and nanofluid dynamics. In the biological tissues and other materials interacting with optical radiation, those terms determining additional sources of ballistic heat production become important [1].

Solution of (19) with the boundary and initial conditions (20)-(22) will be obtained and studied. The corresponding solutions of (18) and (17) with the same boundary conditions can be obtained then by assuming $K n=0$ , $q^{\prime}=0$ in (18), and additionally $\tau^{0}=0$ in (17).

The heat equation with GK law has already been studied for solid materials [25], but the bioheat equations (1), (3), (9) with temperature dependent heat sources and boundary conditions (14)-(16) have not been properly studied yet. The analytical solution for the temperature independent heat sources has been studied in [13].

Solution of (19) can be found in the form

$$
\mathrm{T}(t, x)=\Theta(t, x)+J_{1}^{0}(t) \mathrm{X}_{1}(x)+J_{2}^{0}(t) \mathrm{X}_{2}(x)
$$

where $\Theta(t, x)$ satisfies the following PDE and boundary conditions

$$
\begin{gathered}
\tau^{0} \frac{\partial^{2} \Theta}{\partial t^{2}}+\mathrm{A}_{1} \frac{\partial \Theta}{\partial t}-\mathrm{A}_{2} \frac{\partial^{3} \Theta}{\partial t \partial x^{2}}-\mathrm{A}_{3} \frac{\partial^{2} \Theta}{\partial x^{2}}+\mathrm{A}_{4} T=q-F(t, x) \\
x=0: \frac{\partial \Theta}{\partial x}=0 \\
x=1: \frac{\partial \Theta}{\partial x}=0 \\
t=0: \quad \Theta=-J_{1}^{0}(0) X_{1}(x)-J_{2}^{0}(0) X_{2}(x)=0
\end{gathered}
$$

where $F(t, x)=\sum_{k=1}^{2} \tau^{0} J_{k}^{0 / \prime} X_{k}-A_{3} J_{k}^{0} X_{k}^{\prime \prime}+A_{4} J_{k}^{0} X_{k}, \mathrm{~A}_{1}=1+\tau^{0}\left(\chi-q^{\prime}\right), \mathrm{A}_{2}=K n^{2}, \mathrm{~A}_{3}=\alpha^{0}-K n^{2} \chi, \mathrm{A}_{4}=\chi-q^{\prime}$, stroke sign means the derivative of the functions with respect to their arguments. 
Journal of Thermal Engineering, Research Article, Vol. 5, No. 6, Special Issue 10, pp. 149-161, December, 2019

The functions $\mathrm{X}_{1,2}(x)$ satisfy the system of ODE and boundary conditions

$$
\begin{gathered}
\mathrm{X}_{1,2}^{\prime \prime}=\zeta \mathrm{X}_{1,2} \\
x=0: X_{1}^{\prime}=1, \quad X_{2}^{\prime}=0 \\
x=1: \quad X_{1}^{\prime}=0, \quad X_{2}^{\prime}=1
\end{gathered}
$$

where $\zeta=\mathrm{A}_{1} / A_{2}=\left(1+\tau^{0}\left(\chi-q^{\prime}\right)\right) / \mathrm{Kn}^{2}$.

Solution of (28)-(30) can be easily found. Depending on sign of $\zeta$ two cases must be considered.

Case $1 a$. $\zeta>0$ or $q^{\prime}<\chi+1 / \tau^{0}$. Then,

$$
\mathrm{X}_{1}(x)=-\sqrt{\zeta}^{-1}(\operatorname{cth}(\sqrt{\zeta}) \operatorname{ch}(\sqrt{\zeta} x)-\operatorname{sh}(\sqrt{\zeta} x)), \quad \mathrm{X}_{2}(x)=\frac{\operatorname{ch}(\sqrt{\zeta} x)}{\sqrt{\zeta} \operatorname{sh}(\sqrt{\zeta})}
$$

Case $2 a . \zeta<0$ or $q^{\prime}>\chi+1 / \tau^{0}$. Then,

$$
\mathrm{X}_{1}(x)=\sqrt{\frac{1}{\zeta}}(\operatorname{ctg}(\sqrt{\zeta}) \cos (\sqrt{\zeta} x)+\sin (\sqrt{\zeta} x)), \quad \mathrm{X}_{2}(x)=-\frac{\cos (\sqrt{\zeta} x)}{\sqrt{\zeta} \sin (\sqrt{\zeta})}
$$

Case 3a. $\zeta=0$ or $q^{\prime}=\chi+1 / \tau^{0}$. Then (28) has no solutions satisfying (29), (30).

Now $F(t, x)$ can be computed and solution of (24)-(27) can be found for each of the cases (31)-(32). Due to the boundary conditions (25)-(26), the solution can be found as Fourier expansion

$$
\Theta(t, x)=\sum_{n=0}^{\infty} \vartheta_{n}(\mathrm{t}) \cos (\pi n \mathrm{x})
$$

Then, $\vartheta_{k}(\mathrm{t})$ can be found from the equation

$$
\tau^{0} \vartheta_{n}^{\prime \prime}+\left(\mathrm{A}_{1}-\pi^{2} n^{2} \mathrm{~A}_{2}\right) \vartheta_{n}^{\prime}+\mathrm{A}_{4}-\pi^{2} \mathrm{n}^{2} \mathrm{~A}_{3}=\xi_{n}(t)
$$

where $\xi_{n}(t)=\int_{0}^{1}(q-F(t, x)) \cos (\pi n x) d x$.

The fundamental solution of (34) is determined by the sign of the expression $D_{n}=\left(\mathrm{A}_{1}-\pi^{2} \mathrm{n}^{2} \mathrm{~A}_{2}\right)^{2}-4 \tau^{0}\left(\mathrm{~A}_{4}-\pi^{2} \mathrm{n}^{2} \mathrm{~A}_{3}\right)$, and different cases have to be studied.

Case 1b. $D_{n}>0$. Then,

$$
\theta_{n}(\mathrm{t})=C_{1 n} e^{-\lambda_{1 n} t}+C_{2 n} e^{-\lambda_{2 n} t}+\Xi_{n}^{1}(\mathrm{t})
$$

where $\lambda_{1 n, 2 n}=\frac{-\left(\mathrm{A}_{1}-\pi^{2} \mathrm{n}^{2} \mathrm{~A}_{2}\right) \pm \sqrt{D_{n}}}{2 \tau^{0}}, \Xi_{n}^{1}(t)=e^{\lambda_{1 n} t} \int_{0}^{t} e^{-\lambda_{1 n} \varsigma} \xi_{n}(\varsigma) d \varsigma-e^{-\lambda_{2} t} \int_{0}^{t} e^{\lambda_{2 n} \varsigma} \xi_{n}(\varsigma) d \varsigma$.

Case 2b. $D_{n}<0$. Then, 


$$
\theta_{n}(\mathrm{t})=C_{3 n} e^{-\lambda_{3 n} t} \cos \left(\lambda_{4 n} \mathrm{t}\right)+C_{4 n} e^{-\lambda_{3 n} t} \sin \left(\lambda_{4 n} \mathrm{t}\right)+\Xi_{n}^{2}(\mathrm{t})
$$

where $\lambda_{3 n}=\frac{\pi^{2} \mathrm{n}^{2} \mathrm{~A}_{2}-\mathrm{A}_{1}}{2 \tau^{0}}, \lambda_{4 n}=\frac{\sqrt{D_{n}}}{2 \tau^{0}}$

$\Xi_{n}^{2}(t)=\frac{e^{-\lambda_{3 n} t}}{\lambda_{4 n}}\left[\sin \left(\lambda_{4 n} \mathrm{t}\right) \int_{0}^{t} e^{\lambda_{3 n} \varsigma} \cos \left(\lambda_{4 n} \varsigma\right) \xi_{n}(\varsigma) d \varsigma-\cos \left(\lambda_{4 n} t\right) \int_{0}^{t} e^{\lambda_{2 n} \varsigma} \sin \left(\lambda_{4 n} \varsigma\right) \xi_{n}(\varsigma) d \varsigma\right]$.

Case $3 b . D_{n}=0$. Then,

$$
\theta_{n}(\mathrm{t})=\left(C_{5 n}+C_{6 n} t\right) e^{-\lambda_{3 n} t}+\Xi_{n}^{3}(\mathrm{t})
$$

where $\Xi_{n}^{3}(t)=t e^{\lambda_{3 n} t} \int_{0}^{t} e^{-\lambda_{3 n} \varsigma} \xi_{n}(\varsigma) d \varsigma-e^{\lambda_{3} t} \int_{0}^{t} \varsigma e^{\lambda_{3 n} \varsigma} \xi_{n}(\varsigma) d \varsigma$.

The unknown coefficients in (35)-(37) can be found from the initial conditions

$$
t=0: \quad \theta_{n}=0, \quad \theta_{n}^{\prime}=0
$$

as

$$
\begin{aligned}
& C_{1 n}=\frac{\lambda_{2 n} \Xi_{n}^{1}(0)+\Xi_{n}^{1 \prime}(0)}{\lambda_{1 n}-\lambda_{2 n}}, \quad C_{2 n}=-\frac{\lambda_{1 n} \Xi_{n}^{1}(0)+\Xi_{n}^{1 \prime}(0)}{\lambda_{1 n}-\lambda_{2 n}}, \quad C_{3 n}=-\Xi_{n}^{2}(0) \\
& C_{4 n}=\frac{\lambda_{3 n} \Xi_{n}^{2}(0)+\Xi_{n}^{2 \prime}(0)}{\lambda_{3 n} \lambda_{4 n}}, \quad C_{5 n}=-\Xi_{n}^{3}(0), \quad C_{6 n}=-\left(\lambda_{3 n} \Xi_{n}^{3}(0)+\Xi_{n}^{3 \prime}(0)\right)
\end{aligned}
$$

Due to the dependence $D_{n}(n)$ all the cases $1 \mathrm{~b}-3 \mathrm{~b}$ can be found in the set of solutions $\left\{\theta_{n}(\mathrm{t})\right\}_{n=1}^{N}$.

\section{RESULTS AND DISCUSSION}

Numerical computations of the heat propagation across a layer of biological material with distributed positive (chemical reactions) and negative (carrying away by blood flow) sources have been carried out on (23), (31)-(33), (35)-(37). The following model parameters correspondent to human skin, muscle and bone tissues [1, 11, 13, 20, 27] have been used:

1) Common parameters: $T_{b}=37^{\circ} \mathrm{C}, \rho_{b}=1160 \mathrm{~kg} / \mathrm{m}^{3}, c_{b}=3650-3770 \mathrm{~J} / \mathrm{kg} \cdot \mathrm{K}, w_{b}=0.5-3 \mathrm{~kg} / \mathrm{m}^{3} \cdot \mathrm{s}$.

2) Skin: $\quad h=0.25-2.5 \mathrm{~cm}, \quad \rho=1190 \mathrm{~kg} / \mathrm{m}^{3}, \quad c=3600-3768 \mathrm{~J} / \mathrm{kg} \cdot \mathrm{K}, \quad \lambda=0.235-0.42 \mathrm{~W} / \mathrm{m} \cdot \mathrm{K}$, $q_{\text {met }}=245-368 \mathrm{~W} / \mathrm{m}^{3}, \tau=0.001 \mathrm{~s}$.

3) Muscle: $\quad h=0.5-3.5 \mathrm{~cm}, \quad \rho=1190 \mathrm{~kg} / \mathrm{m}^{3}, \quad c=3600-3768 \mathrm{~J} / \mathrm{kg} \cdot \mathrm{K}, \quad \lambda=0.44-0.5 \mathrm{~W} / \mathrm{m} \cdot \mathrm{K}$, $q_{\text {met }}=368-684 \mathrm{~W} / \mathrm{m}^{3}, \tau=0.4-0.5 \mathrm{~s}$.

4) Bone: $\quad h=0.35-1 \mathrm{~cm}, \quad \rho=1190 \mathrm{~kg} / \mathrm{m}^{3}, \quad c=3600-3768 \mathrm{~J} / \mathrm{kg} \cdot \mathrm{K}, \quad \lambda=0.17-0.19 \mathrm{~W} / \mathrm{m} \cdot \mathrm{K}$, $q_{\text {met }}=220-300 \mathrm{~W} / \mathrm{m}^{3}, \tau=1-1.5 \mathrm{~s}$.

The physiological limits of the parameters are determined by individual variations. Other parameters are determined by the frequency of optical radiation and the chemical reactions induced by it. Therefore, the sets of nondimensional values for $q_{\text {met }}^{0}, q_{\text {rad }}^{0}, \lambda_{\text {met }}^{0}, \lambda_{\text {rad }}^{0} \in[0,1]$ have been tested. The numerical computations have been carried out for $n=10$ harmonics; that provides fast convergence of the series with accuracy $\sim 10^{-5}$. The typical dynamics of the temperature curves is similar to the skin, muscle and bone tissues with different rates of the temperature rise and 
Journal of Thermal Engineering, Research Article, Vol. 5, No. 6, Special Issue 10, pp. 149-161,

December, 2019

relaxation determined by the thermal properties of the tissue. Since in the live tissues the noninvasive surface temperature measured over the skin surface is only available, the numerical results presented in Figure 3-5 have been computed for the skin parameters.

The computed temperature curves $\mathrm{T}(\mathrm{t})$ during the heating and thermal relaxation are depicted in Figure 3a-c in the dimensional form for different tissue models. The Fourier heat conduction law based bioheat equation (17) describes an exponential temperature rise during the heating and single time exponential temperature decay during the thermal relaxation at different depths of the layer (Figure 3a). The decay is quite slow so the depth of penetration of the irradiation is overestimated when the model (17) is used [4]. The modified model (18) better describes the complex dynamics of temperature rise with a plateau (Figure 3b), that closer corresponds to the experimental data (Figure 2) than the classical Pennes model (Figure 1). The model (19) correctly describes not only the temperature growth but also its thermal relaxation with two characteristic exponents corresponding to the solutions (35), (38) depending on the model parameters.

The temperature distributions in the same layer modeled by (18), (19) at different intensity of heating are presented in Figure 4.
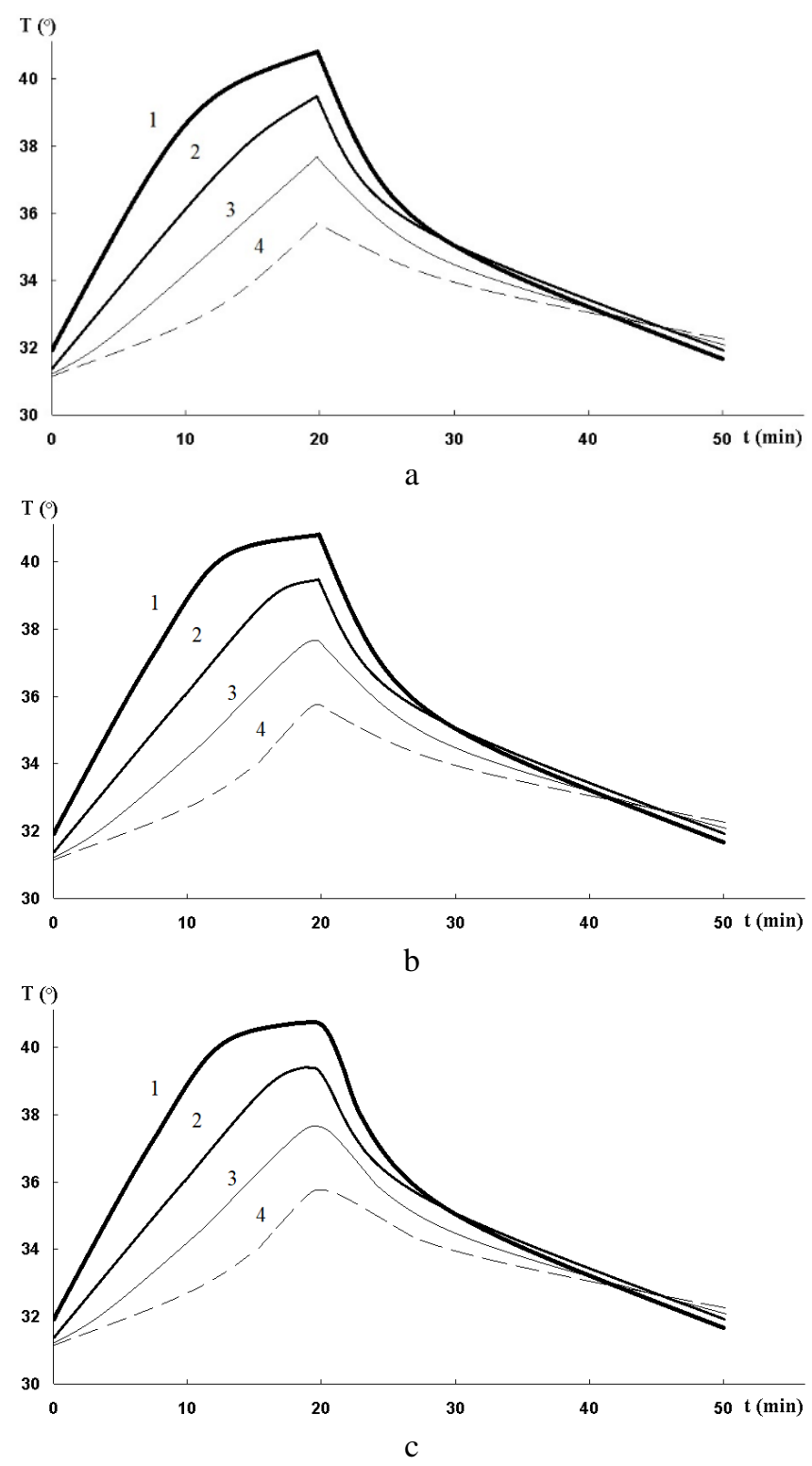

Figure 3. Computed curves $\mathrm{T}(\mathrm{t})$ on (a) the equation (17), (b) equation (18), (c) equation (19), where the curves 1-4 correspond to different depths $\mathrm{h} / \mathrm{L}=0.25 ; 0.5 ; 0.75 ; 0.95$ of the layer 
Journal of Thermal Engineering, Research Article, Vol. 5, No. 6, Special Issue 10, pp. 149-161, December, 2019
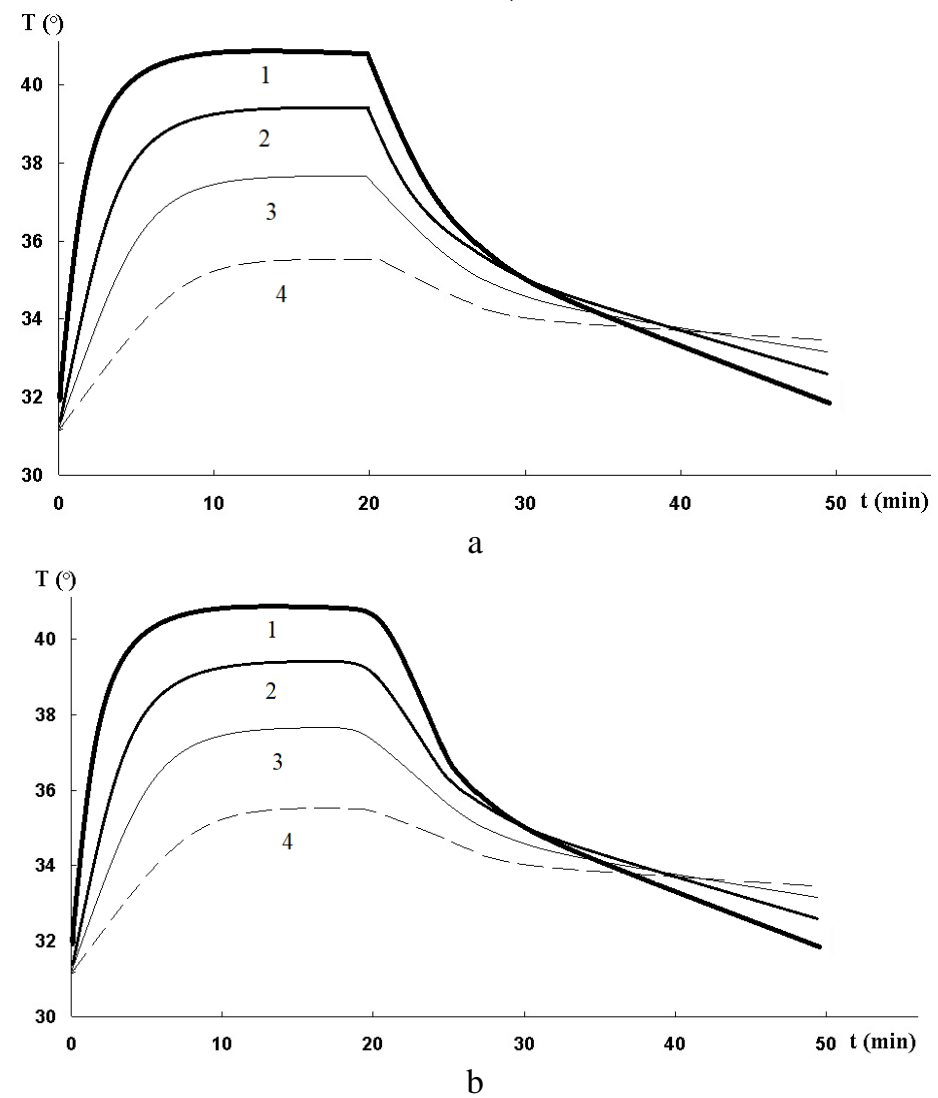

Figure 4. The temperature $\mathrm{T}(\mathrm{t})$ curves for $20 \mathrm{~min}$ heating followed by 30 min thermal relaxation computed on (a) the model (18), and (b) model (19), at $J_{2}^{0}=0$; the curves $1-4$ correspond to $J_{1}^{0}=4 ; 3 ; 2 ; 1$ accordingly

The maximal values of the temperatures at the plateau (Figure 3c, Figure $4 b$ ) are determined by individual thickness $h$, intensity of microcirculation $w_{b}$ and metabolic heat production $q_{\text {met }}$. The thermal parameters of the tissues are more or less stable for different individuals and even in mammals [27]. Therefore, the computed solution allows identification of two individual parameters $\left(w_{b}, q_{\text {met }}\right)$, while the thickness of skin and underlying tissues can be estimated by palpation [1]. Based on the individual $\left(w_{b}, q_{\text {met }}\right)$ data, the patient specific safe dose of irradiation allowing the LLL or LED therapy without uncomforting or damaging temperatures in the treated tissues.

Comparative results on numerical computations of the temperature curves $\mathrm{T}(\mathrm{t})$ by the model with and without accounting for the distributed heat sources with corresponding experimental data obtained on the skin (Figure 5a), muscle (Figure 5b) and bone (Figure 5c) tissues with different $w_{b}, q_{\text {met }}$ and the tissue parameters identified on the previous step on the averaged curves shows significant qualitative differences between two models. The most essential differences are marked in Figure 5 by arrows. The model without heat sources exhibited purely exponential temperature rise during the heating period and sudden exponential decay immediately after the heating had been stopped. It is single-time relaxation and the temperature decays exponentially and could reach low non-physiological values even at some realistic times $\mathrm{t} \sim 1.5-2 \mathrm{~h}$. When the hear sources activated by the photoinduced reactions are accounted for, the temperature rise (arrows 1 in Figure 5a-c) is not such acute as the exponential one and it is closer to the measurement data for all the surface tissues studied. The temperature decay clearly demonstrates two relaxation times: initial fast relaxation (arrows 2 in Figure 5a-c) provided by absence of the photoinduced heating due to the photoinduced exothermal biochemical reactions and activation of the microcirculation, but the transition to the relaxation is not sharp as in the exponential model because of the inertia of both biochemical processes and regulatory reactions of the activated microcirculation. The second phase of the relaxation is characterized by slow relaxation that produces high surface temperatures at $\mathrm{t}=50 \mathrm{~s}$ than by the exponential relaxation due to presence of distributed metabolic heat sources, which maintains the surface temperatures on the physiological level close to the initial surface 
Journal of Thermal Engineering, Research Article, Vol. 5, No. 6, Special Issue 10, pp. 149-161, December, 2019

temperature before the experiment evet at $\mathrm{t}>2 \mathrm{~h}$ that correspond to the steady state of metabolism of the skin and other surface tissues.
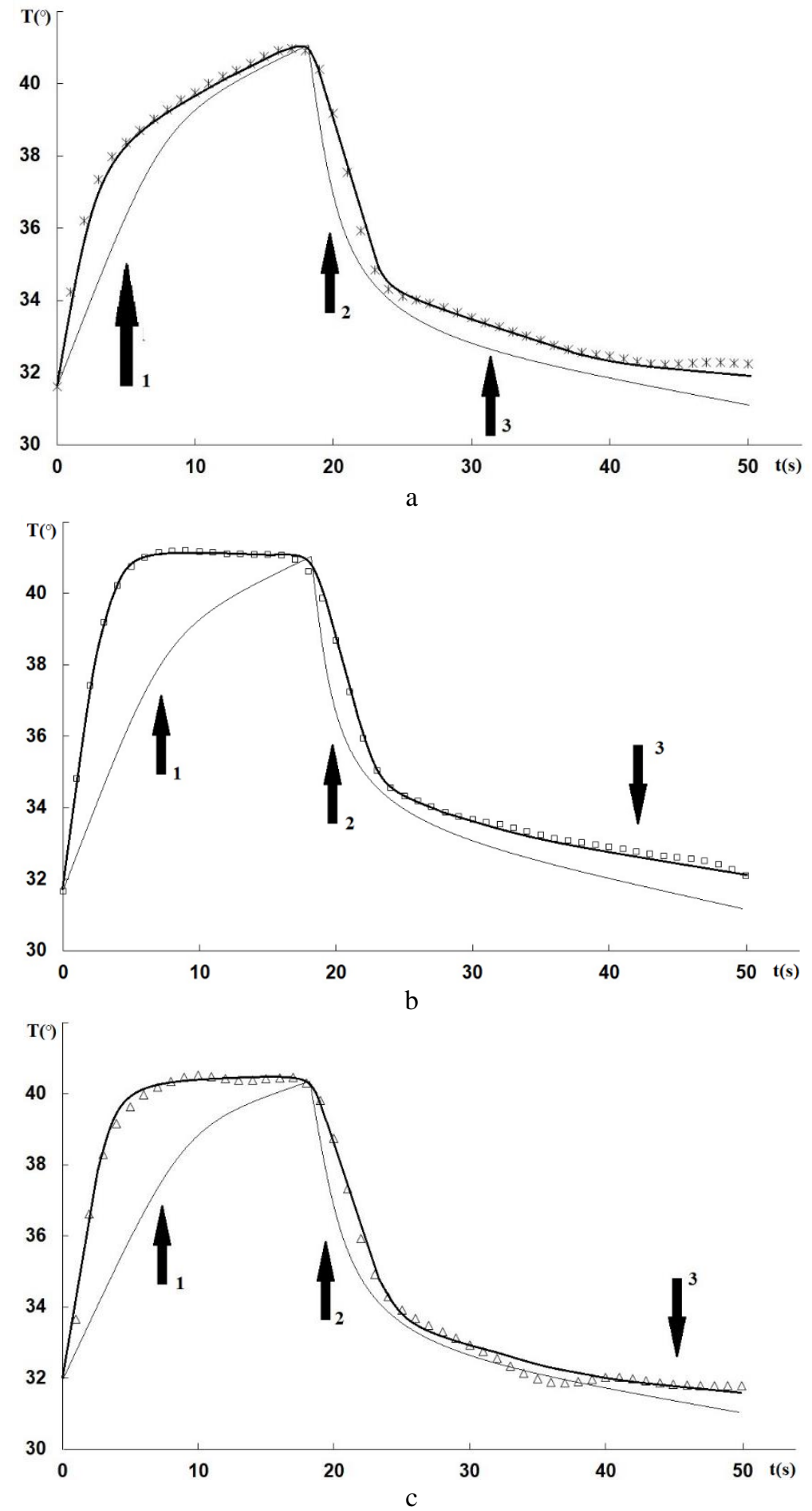

Figure 5. Temperature curves $\mathrm{T}(\mathrm{t})$ for (a) skin, (b) muscle and (c) bone tissues computed on the model with (thick lines) and without (thin lines) distributed heat sources, and measured on a volunteer (marked)

\section{CONCLUSIONS}

The heat conduction in the biological tissues is correctly described by the Pennes bioheat equation which accounts for both heat generation in the live tissues and heat removal by the circulating blood. Both phenomena are influenced by 
Journal of Thermal Engineering, Research Article, Vol. 5, No. 6, Special Issue 10, pp. 149-161, December, 2019

the external heating of the tissues by LLL or LED with therapeutic purposes. Nevertheless, the basic heat propagating law included into the

Pennes bioheat equation influence significantly the dynamics of the heat rise and decay during the heating and thermal relaxation of the tissue accordingly. Comparative analysis of the theoretical computed curves to the measured ones reveals the better correspondence of the G-K heat propagation las in the biological tissues. When the heat relaxation time is included into the model, the complex two-time temperature growth is correctly described, but the relaxation decay is oversimplified. When the additional ballistic-type heat propagation is accounted for, the realistic two-time heat decay curves during the thermal relaxation are described correctly. The comparative analysis of the theoretical and measured curves is essential for identification of the individual tissue parameters. Those parameters can be used for quantitative estimations of the safe therapeutic dose of the irradiation based on the computations of the damaging parameter [13] that will be a subject of further studies.

\section{REFERENCES}

[1] Grossweiner, L. I. (2005). The Science of Phototherapy: An Introduction. Springer Science \& Business Media.

[2] Gomer, Ch. J. (2010) Photodynamic Therapy: Methods and Protocols. Humana Press.

[3] Kizilova, N., \& Korobov, A. (2018). On biomedical engineering techniques for efficient phototherapy. International Journal on Biosensors and Bioelectronics, 4(6), 289-295.

[4] Kizilova, N., \& Korobov, A. (2016). Mechanisms of influence of the low-intense optical radiation on the microcirculatory system. A review. Photobiology and Photomedicine, 71(2), 79-93.

[5] Liu, K.-C. (2008). Thermal propagation analysis for living tissue with surface heating. International Journal of Thermal Sciences, 47, 507-513.

[6] Kumar, P., Kumar, D., Rai, K. N. (2015). A numerical study on dual-phase-lag model of bio-heat transfer during hyperthermia treatment. Journal of Thermal Biology, 49-50, 98-105.

[7] Ciesielski, M., Mochnacki, B., Piasecka-Belkhayat, A. (2016). Analysis of temperature distribution in the heated skin tissue under the assumption of thermal parameters uncertainty. 40th Solid Mechanics Conference, Book of Abstracts, Warsaw, IPPT, P048.

[8] Verma, S. K., Maheshwari, S., Singh, R. K., Chaudhari, P. K. (2012). Laser in dentistry: An innovative tool in modern dental practice. National Journal of Maxillofacial Surgery, 3(2), 124-132.

[9] Azadgoli, B., Baker, R. Y. (2016). Laser applications in surgery. Annals of Translational Medicine, 23(4), 452458.

[10] Pennes, H. H. (1948). Analysis of tissue and arterial blood temperatures in the resting forearm. Journal f Applied Physiology, 1, 93-122.

[11] Xu, F., Lu, T. J., Seffen, K. A. (2008). Biothermomechanics of skin tissues. Journal of the Mechanics and Physics of Solids, 56, 1852-1884.

[12] Nóbrega, S., Coelho, P. J. (2017). A parametric study of thermal therapy of skin tissue. Journal of Thermal Biology, 63, 92-103.

[13] Lin, S.-M. (2013). Analytical solutions of bio-heat conduction on skin in Fourier and non-Fourier models. Journal on Mechanics in Medicine and Biology, 13 (4), 1350063.

[14] Kizilova, N., Korobov, A., Solovjova H. (2018). Heat transfer at local surface freezing of human tissues: experimental and theortical study. In: IX Internal Conference "Low temperature physics," Abstracts book, Kharkiv, Ukraine, 148.

[15] Kizilova N., Korobov A. (2018). On bioheat equation and its modification. In: 3-rd International Conference "Differential equations and Control Theory," Book of Abstracts, Kharkiv, Ukraine, 30-31.

[16] Tzou, D. Y. (1995). A unified field approach for heat conduction from macro- to microscales. Journal of Heat Transfer, 117(1), 8-16.

[17] Tzou, D. Y. (1997). Macro- to Microscale Heat Transfer: The Lagging Behavior, New York, Taylor and Francis.

[18] Cattaneo, C. (1948). Sulla conduzione del calore, Atti del Seminario Matematico e Fisico dell' Universita di Modena, 3, 83-101.

[19] Vernotte, P. (1958). Les paradoxes de la théorie continue de l'équation de la chaleur. Comptes rendus hebdomadaires des séances de l'Académie des sciences, 46, 3154-3155. 
Journal of Thermal Engineering, Research Article, Vol. 5, No. 6, Special Issue 10, pp. 149-161, December, 2019

[20] Majchrzak, E., Turchan, Ł., Dziatkiewicz, J. (2015). Modeling of skin tissue heating using the generalized dual phase-lag equation. Archives of Mechanics, 67(6), 417-437.

[21] Rukolaine, S. A. (2014). Unphysical effects of the dual-phase-lag model of heat conduction. International Journal of Heat and Mass Transfer, 78, 58-63.

[22] Rukolaine, S. A. (2017). Unphysical effects of the dual-phase-lag model of heat conduction: higher-order approximations. International Journal of Thermal Sciences, 113, 83-88.

[23] Wang, M., Yang, N., Guo, Z.-Y. (2011). Non-Fourier heat conductions in nanomaterials. Journal of Applied Physics, 110(6), 064310.

[24] Kovács, R. (2017). Heat conduction beyond Fourier's Law: theoretical predictions and experimental validation, Budapest University of Technology and Economics, Ph.D. Thesis.

[25] Ván, P., Berezovski, A., Fülöp, T., Gróf, G., Kovács, R., Lovas, Á., \& Verhás, J. (2017). Guyer-Krumhansl-type heat conduction at room temperature. Europhysics Letters, 118, 50005.

[26] Tang, D. W., Araki, N. (2000). Non-Fourier heat condution behavior in finite mediums under pulse surface heating. Material Science Engineering A, 292, 173.

[27] Berezovski, V. A., Kolotylov, N. N. (1990). Biophysical characteristics of human tissues: A reference book, Kiev, Naukova Dumka. 